\title{
Tools of the trade: the bio-cultural evolution of the human propensity to trade
}

\author{
Armin W. Schulz ${ }^{1}$ iD \\ Received: 28 August 2021 / Accepted: 16 February 2022 / Published online: 3 March 2022 \\ (c) The Author(s), under exclusive licence to Springer Nature B.V. 2022
}

\begin{abstract}
Humans are standouts in their propensity to trade. More specially, the kind of trading found in humans-featuring the exchange of many different goods and services with many different others, for the mutual benefit of all the involved parties - far exceeds anything that is found in any other creature. However, a number of important questions about this propensity remain open. First, it is not clear exactly what makes this propensity so different in the human case from that of other animals. Second, it is not clear why other animals did not acquire this propensity to the extent that humans did. Third, it is not clear what explains the fact that the extent to which humans engage in trade is culturally highly variable. The paper argues that at the heart of the human-animal divergence in this propensity is the particular socio-cultural environment in which humans evolved. This has led them to sometimes, but not always, acquire the cognitive technology (writing, algebra, tallying devices, money, etc.) to support a sophisticated disposition and capacity for reciprocal cooperation, and deep and wide concepts of property and exchange value.
\end{abstract}

Keywords Cooperation · Exchange · Gene-culture coevolution · Market · Property · Technology $\cdot$ Trade $\cdot$ Tool

\section{Introduction}

Humans are cognitively unique in many different ways. They are exceptional cultural learners and mindreaders, they use and manufacture highly complex tools, and they rely on highly abstract and complex mental and linguistic representations in interacting with their environment (Tomasello 1999; Heyes 2018; Boyd and Richerson 2005; Henrich 2015; Sterelny 2012; Vaesen 2012; Osiurak and Reynaud, forthcoming). Furthermore - and, as will become clearer below, not unrelatedly-humans are

Armin W. Schulz

awschulz@ku.edu

http://people.ku.edu/ a382s825/

1 Department of Philosophy, University of Kansas, 3101 Wescoe Hall, Lawrence, KS 66045, USA 
standouts in their "propensity to truck, barter and exchange" (Smith 1776; see also Ofek 2001; Ofek 2013; Nowell 2010). That is, humans are at least sometimes disposed to exchange many different goods and services with many different others, for the mutual benefit of all the involved parties.

The fact that humans are standout traders has been known for a while. So, Smith (1776 I, ii) notes that:

The division of labour, from which so many advantages are derived, is not originally the effect of any human wisdom, which foresees and intends that general opulence to which it gives occasion. It is the necessary, though very slow and gradual consequence of a certain propensity in human nature which has in view no such extensive utility; the propensity to truck, barter, and exchange one thing for another.

Whether this propensity be one of those original principles in human nature of which no further account can be given; or whether, as seems more probable, it be the necessary consequence of the faculties of reason and speech, it belongs not to our present subject to inquire. It is common to all men, and to be found in no other race of animals, which seem to know neither this nor any other species of contracts. [...] Nobody ever saw a dog make a fair and deliberate exchange of one bone for another with another dog. Nobody ever saw one animal by its gestures and natural cries signify to another, this is mine, that yours; I am willing to give this for that. [...] But man has almost constant occasion for the help of his brethren, and it is in vain for him to expect it from their benevolence only. He will be more likely to prevail if he can interest their selflove in his favour, and show them that it is for their own advantage to do for him what he requires of them. Whoever offers to another a bargain of any kind, proposes to do this. Give me that which I want, and you shall have this which you want, is the meaning of every such offer; and it is in this manner that we obtain from one another the far greater part of those good offices which we stand in need of. It is not from the benevolence of the butcher, the brewer, or the baker that we expect our dinner, but from their regard to their own interest. We address ourselves, not to their humanity but to their self-love, and never talk to them of our own necessities but of their advantages.

For present purposes, the key aspect of this passage is that Smith contrasts the ways human and non-human animals go about getting what they want. Whereas the latter simply fight over the possession of a scarce resource, humans decide to barter over it. Humans as well as dogs (say) sometimes have opposing interests and are not always altruistically inclined towards each other; however, it is distinctive of humans that they often resolve these conflicts through trade rather than fighting. ${ }^{1}$

\footnotetext{
${ }^{1}$ Human socio-cultural evolution also allowed for a greater diversity of preferences, creating more winwin situations and thus making it easier to resolve conflicts through trade. This is an important point to which the paper returns in Sect. "The bio-cultural evolution of the human propensity to trade".
} 
However, while thus commented on for quite some time, a number of important questions about this human propensity to trade remain open. ${ }^{2}$ First, as it stands, it is not clear exactly what makes this propensity so different in the human case from that of other animals. Exactly how do humans differ from other animals in their cognitive and motivational abilities when it comes to trade? Second, it is not clear why humans evolved in this way. Why did other animals not acquire the propensity to trade (or at least not to the extent that humans did)? What unique evolutionary pressures in the human lineage led to this divergence? ? $^{3}$ Third, it is not clear what explains the fact that the extent to which humans engage in trade is culturally highly variable-with some cultures (such as contemporary Western society) seeing a lot of trade, and some (such as some contemporary hunter-gatherer cultures like the Hadza) seeing less trade. Given that all humans could engage in extensive trade, why don't they all do so? Answering these questions is the goal of the rest of this paper.

The paper is structured as follows. In section II, the propensity to trade is shown to be divisible into a number of component abilities. In section III, I then contrast and make more precise the extent to which humans differ from non-human animals in these component abilities. In section IV, I provide an account of the evolutionary pressures that have led humans to diverge from non-human animals in their propensity to trade. I conclude in section V.

\section{The Propensity to Trade}

In the passage quoted earlier, Smith (1776) suggests that the core of the distinction between humans and dogs in their propensity to trade lies in the sophistication of their egoism. While both individual humans and individual dogs are egoistic in the sense of looking to get the best for themselves without an inherent ("ultimate"Sober and Wilson 1998; Stich 2007; Schulz 2011) concern for the interests of others, humans recognize that others have their own interests, and that mutually beneficial outcomes can be achieved through trade rather than all-out fighting. ${ }^{4}$

This is a useful start towards characterizing the propensity to trade, and it does get at several important aspects of this propensity. (Here and in what follows, I use the terms "propensity to truck, barter, and exchange" and "propensity to trade" interchangeably.) However, it also turns out that this characterization needs to be made more precise in several regards. Indeed, the propensity to trade should be seen to have three key psychological components that underlie it: (1) a concept of property, (2) a concept of exchange value, and (3) a disposition and capacity for reciprocal

\footnotetext{
${ }^{2}$ For an expanded treatment especially of the evolutionary consequences of the propensity to trade, see Ofek (2001). See also Avsar (2020).

${ }^{3}$ This evolutionary perspective was, of course, not as such available to Smith (1776); but see also Schliesser (2011).

${ }^{4}$ Note that Smith (1776) does not claim that dogs or humans have no altruistic motivations whatsoever. His point is just that they have egoistic motivations that frequently influence their behavior. See also Schulz (2018).
} 
cooperation (Brosnan 2011; Brosnan and Beran 2009; Avsar 2020; Ofek 2001). ${ }^{5}$ Of course, these three psychological components do not fully exhaust what is needed to have a propensity to trade. In particular, trading also requires basic communicative abilities (to make clear to others what is to be traded for what), basic theory of mind abilities (to determine whether others are willing to engage in trade), and basic abilities to evaluate different possible states of the world (to assess whether trading for a resource is called for in the situation at hand).

However, the three psychological capacities of a concept of property, a concept of exchange value, and a disposition and capacity for reciprocal cooperation are especially central to the propensity for trade. On the one hand, organisms that have these abilities are very likely to have the remaining other capacities as well. ${ }^{6}$ For example, in order to be capable and disposed to cooperate reciprocally, basic mindreading and decision-making abilities are needed (Mulcahy and Call 2006; Kabadayi and Osvath 2017) —indeed, trading is, as will be made clearer below, a specific subtype of reciprocally cooperative interactions. ${ }^{7}$ On the other hand, organisms with even strongly developed abilities of communication, mindreading, and decision-making, without the concepts of property and exchange value and the disposition and capacity for reciprocal cooperation do not have the psychological underpinnings for being trader. Organisms that do not recognize ownership and transfer of ownership-even in minimal way-and / or which are not disposed towards reciprocal cooperation, cannot exchange goods and services with each other, no matter how good they are in communicating, reading each other's minds or evaluating different possible future states of the world. (That said, as will be made clearer in section IV below, these other abilities may be crucial components in underwriting the bio-cultural evolution of an extensive disposition to trade.) With this in mind, consider the three core components of the propensity to trade in more detail.

First, in order to recognize that mutually beneficial outcomes can be achieved through trade, an organism needs to have a concept of property. Trade-at least in the sense that is relevant here-concerns the exchange of owned goods and services. For two organisms to exchange anything, though, they need to be able to recognize something as theirs and other things as belonging to someone else. Common pool resources cannot be exchanged: if I want to drink from the pond you usually drink from, and you want to drink from the one I usually drink from, then we can certainly switch ponds. However, this is really no different from me deciding what to do on my own, without taking into account your situation (and the same goes for you).

\footnotetext{
5 A quick word about trade in services. In a very general sense, trading in services-i.e. the capacity for reciprocal cooperation (3) - is a more foundational ability than a propensity for trade proper. For an organism to truly engage in trading over services, it would need to see itself as owning its actions (cf. Locke, 1987 [1866]). This also converges with the arguments in Ofek (2001) that the provision of fire was foundational in human evolution: fire is an impure public good (it is excludable, but non-rival), and thus facilitated a shift from mere reciprocal cooperation to genuine trading.

6 This is especially so, since highly sophisticated versions of these other abilities are not in fact needed to be a trader: for example, it is a classic economic insight that an agent does not have to be able to attribute complex mental states to others to engage in trade; attributing a disposition to choose in certain ways is sufficient (Hausman, 1992, 2012; Binmore, 2007).

7 See also note 18 below.
} 
Similarly, if I come to your aid in defending against an intruder, in the hopes that you will do the same for me in the future, this may be mutually beneficial and cooperative, but it is not trade. A trade-in the sense that is relevant here-only happens when I recognize that you have a claim on something and I have a claim on something else, and that we can swap these two claims (Ofek 2001, p. 144). ${ }^{8}$

Now, exactly how to characterize the necessary concept of property is not entirely obvious or uncontroversial. However, at a minimum, it requires the recognition that others are excludable from the use of the entity in question: if something $\mathrm{P}$ is the property of some organism $\mathrm{O}$, then $\mathrm{O}$ can prevent others for using or accessing $\mathrm{P}$ (Scorolli et al. 2018; Hartley 2019; Tse 2008; Torii 1974; Sherratt and MestertonGibbons 2015; Tibble and Carvalho 2018). Beyond this minimal requirement, property concepts of different depths and extents can be distinguished.

On the depth side, property concepts are shallower if they see property as anything from which others can be excluded and which can be disposed of at will (without there needing to be a specific justifying reason for the exclusion and use); they gain in depth if property is seen as something from which others can be rightfully excluded and whose use others have no rightful say over (Brosnan 2011; Tse 2008; Torii 1974). ${ }^{9}$ So, if O can simply force others off a piece of land, this land would be seen as O's territory on the former concept, but not necessarily on the latter; on the latter concept, it would need to be determined whether the exclusion can be backed up with sufficient reasons. ${ }^{10}$ (This also implies that theft cannot be distinguished from legitimate property transfer on shallower property concepts, but that it can be so distinguished on the deeper ones.)

As far as extent is concerned, property concepts are wider the more different things they allow to be owned. So, if only territory is recognized as ownable, this is a narrower property concept than one that would also allow for foodstuffs and tokens of exchange ("money") to be ownable. I return to these points in the next section, but for now, it is just important to note that the concept of property is intertwined with the ability to recognize (more or fewer) things as being claimed (with or without an explicitly represented reason). ${ }^{11}$

\footnotetext{
${ }^{8}$ Ofek (2001, p. 144) suggests that pure private goods are unlikely to make the basis for trade, but it is not clear why: while individual tokens of private goods cannot be divided, they can still be traded. At any rate, this issue is not central here.

${ }^{9}$ Alternatively and equivalently, one can mark this distinction as concerning "temporary" or "permanent" ownership (Scorolli et al., 2018). Note also that it is possible to further distinguish things from which one can exclude others and things of which one can dispose at will (cf. Native title to land in Australia). I thank an anonymous referee for useful discussion of this point.

10 The depth of the concept can be said to be increasing with the quantity and complexity of the needed reasons - though making this precise is not necessary for present purposes.

11 The two dimensions of depth and extent are at least logically independent of each other (though they may be correlated with each other for non-logical reasons). Note also that the distinction between property concepts of different degrees of depth and breadth is sometimes marked by distinguishing concepts of ownership, possession, and territoriality (Blake \& Harris, 2009; Kanngiesser et al., 2020). However, this latter phrasing seems to suggest that this distinction is one of kind, whereas it is more plausibly seen as one of degree. At any rate, this is merely a verbal issue.
} 
Second, the propensity to trade must be seen to be underwritten by a concept of exchange value (Avsar 2020, pp. 63-64). For an organism to exchange something A for something $\mathrm{B}$, these two things have to be conceived of as exchangeable in the first place. This need not mean that A and B need to be seen as of the same value for either one or all parties of the exchange. ${ }^{12}$ Rather, the point is that trades ranges over items that are conceived of as having a comparable "trade identity." This trade identity need not be the same as an organism's individuation schema for what the world is like: organisms are not forced to see grapes and cucumbers as exchangeableand certainly not as exchangeable one-to-one-even though they might recognize that the world divides into cucumbers and grapes (among other things) (Brosnan et al. 2008; Oberliessen and Kalenscher 2019). What is needed is that organisms recognize that their claims on various things can be transferred to other people-and vice-versa.

Accordingly, concepts of exchange value can be wider and narrower. They are wider, if more things can be exchanged for each other, with complete comparability being the extreme case. They are narrower if only some things can be so compared (i.e. if some things do not have an exchange value). Note that organisms may have a relatively narrow concept of exchange value and a relatively wide concept of property: many things can be owned, but only few things can be traded. The key is just that the ability to trade requires that things can be seen as changing owners.

The third component of the propensity to trade is the one Smith (1776) focused on: the disposition and capacity to cooperate reciprocally (Avsar 2020, pp. 52, 60-63, 90-95). The benefits from trade might accrue a long time after the trading itself has taken place. In the short term, I may (and generally do) gain more from just taking whatever it is that I want of yours-and keeping whatever I have. However, trading now may make future trades possible, with the overall benefit of trading outweighing the short-term benefits of aggression (Ofek 2001; Cosmides and Tooby 1992). Indeed, the benefits of trade may not even involve the other trading party at all. If A trades with B-instead of just taking whatever it wants of B's-A may make itself more attractive as a future trading partner of $\mathrm{C}$. If $\mathrm{C}$ has resources that $\mathrm{A}$ could not obtain otherwise, this can make trade with B indirectly adaptive-even if is not directly so adaptive (Nowak and Sigmund 2005; Hammerstein and Noe 2016; Noe and Voelkl 2013). An extreme form of the latter case is where trading yields a benefit to everyone in the organism's in-group—e.g. by keeping infighting down (Sober and Wilson 1998; Wilson et al. 2013).

Organisms somehow need to be sensitive to these future benefits in order to be traders. If organisms are just motivated by the immediate, direct benefits on offer, they will in general not be motivated to trade. ${ }^{13}$ There are many reasons for why

\footnotetext{
12 This also implies that there is somewhat of a continuum between trading and mere taking. A forced trade-an offer that cannot be refused-is not really a trade at all. Less pronounced, but still uneven power relationships make for borderline cases: if a party A has the power to make another party B accept terms of trade that they would otherwise be unwilling to accept, then this is non-paradigmatic case of trade only.

13 What about environments in which seizure is either impossible or carries risk of serious costs (which may have been true for early human social environments)? It is true that, if a party cannot seize the item in question but there is really nothing else that it wants as much, it may be willing to trade something else
} 
this might be so: for example, these organisms may not recognize the potential benefits from trade, or they may recognize them, but not be motivated to act on them. Indeed, they may even be motivated by them, but be unable to resist the pull of the present benefits of aggression (Rosati et al. 2006; Stevens and Stephens 2008; Ofek 2001, p. 142; Avsar 2020, p. 72). Sections III and IV return to some of these reasons; for now, the key point to note is just that, as also pointed out by Smith (1776), the propensity to trade needs to be seen to be underwritten by a robust disposition and capacity to cooperate reciprocally, since the gains from trade need not be immediately obvious and might take a long time to materialize.

All in all, therefore: the propensity to trade is centrally (though not exclusively) underpinned by the conjoint presence of three different psychological and behavioral traits: (a) a concept of property, (b) a concept of exchange value, and (c) a disposition and capacity to cooperate reciprocally. It is important, though, to be clear on the extent of this claim. The idea here is that organisms with a propensity to trade need to have traits (a)-(c). The idea is not that organisms with the abilities (a)-(c) necessarily engage in much trade. Whether the latter claim is true depends on a number of further factors - a point to which section IV returns. For present purposes, what is important to keep in mind is just that abilities (a)-(c) provide the major raw materials out of which the propensity to trade is built.

\section{Human trading}

Are humans extensive traders? In one sense, the answer is clearly yes. As a species, humans trade vast amounts of stuff: in 2018, (nominal) global trade was valued at $\$ 19.48$ trillion and accounted for about $60 \%$ of global GDP (World Bank); while the COVID-19-pandemic has lowered these values significantly, they are unquestionably still very high from the perspective of comparative psychology. While other animals also engage in some forms of trading-for example, chimpanzees, gorillas, orangutans, and bonobos, have been found to engage in the trade of tokens or foodstuffs (Brosnan et al. 2008; Parrish et al. 2013; Pelé et al. 2009), and cleaner fish and their hosts exchange food for cleaning services (Triki et al. 2019) - there is just no question that humans, as a species, are orders of magnitude more engaged in this activity than other organisms (Ofek 2001; Nowell 2010). That is, as a species, humans are extensive traders, in the sense that some humans trade vast amounts of goods and services with a vast amount of other humans.

However, in another sense, the fact that humans are extensive traders is much less clear. First, the extent to which humans engage in trade is culturally highly variable (Apicella et al. 2014; Hartley 2019; Basu and Waymire 2006, p. 211; Basu et al.

Footnote 13 (continued)

it has now for the item in question. However, for the other party to accept the trade, it still requires that this other party is interested in this other thing (i.e. that it is not just motivated the immediate, direct benefits of the item on offer). I thank an anonymous referee for useful discussion of this point. 
2009). ${ }^{14}$ While trade is a major aspect of life in contemporary Western society, it is less so in other cultures. So, members of the Hadza (or at least those Hadza who are living in more remote areas) tend to engage in little if any trading:

Aside from bows and arrows and the clothes on one's back, few capital goods or personal possessions exist. If one person has more than his or her share of a resource, such as two knives, the surplus will be demanded by others who have less. Thus, to a first approximation, Hadza economic life is characterized by nearly 100 percent taxation and redistribution.

(Apicella et al. 2014, p. 1796). ${ }^{15}$

Similarly, Henrich et al. (2001) note that:

The Machiguenga show the lowest cooperation rates in public good games [in the authors' sample], reflecting ethnographic descriptions of Machiguenga life, which report little cooperation, exchange, or sharing beyond the family unit. (Henrich et al. 2001, p. 76) ${ }^{16}$

This variance in reliance on trade also exists in the temporal dimension: while there are good reasons to think that trade has been a crucial component of the bio-cultural evolution of some human populations (Nowell 2010; Ofek 2001, pp. 176-178, 226-226; 2013; Brooks et al. 2018), the extent to which ancient human cultures relied on trade likely differed across time (Hartley 2019; Avsar 2020). For example, earlier human groups are likely to have relied less on trade, with extensive trading

\footnotetext{
14 Apicella et al. (2014) also show that the Hadza display the endowment effect only in situations where many different things are seen as ownable. This is interesting, as endowment effects have been observed in many different species and for many different goods (Brosnan et al., 2012; Lakshminarayanan et al., 2008; Brosnan et al., 2007; Drayton et al., 2013; Flemming et al., 2012; Kanngiesser et al., 2011; Jaeger et al., 2020). For present purposes, though, it is important to note that endowment effects are not about trade per se, but directly only speak to an organism's reluctance to give up something they have control over. So, Jaeger et al. (2020) and Jones (2001) argue that the endowment effect can be explained as the result of an evolved "time-shifted rationality" (see also Brosnan \& Beran, 2009). The downside of giving up items - especially fitness-relevant ones-is greater than the upside of obtaining them: there is always a chance that a promised return will not materialize, whereas giving up something one already has in possession is a guaranteed loss. Given this, it is possible that organisms display endowment effects without engaging in much trade (when they think that they are unlikely to attain control over a currently uncontrolled item), or that they do not display them even if they do engage in much trade (when they think that they are very likely to obtain control over a currently uncontrolled item). Further discussion of endowment effects is left for a future occasion; for now, though, it is just important to note that the burgeoning literature on endowment effects can be easily combined with the account of the biol-cultural evolution of the propensity to trade set out here.

15 The Hadza can of course still be seen as reciprocators; they just don't trade in the narrow sense relevant here. See also note 5 .

16 However, see also Henrich (1997), which draws a slightly different picture of life among the Machiguenga (at least to the extent they are connected to Western trading institutions). However, whatever exactly may be true about the Machiguenga, the general point in the text is not controversial: the extent to which humans engage in trade is culturally highly variable.
} 
institutions only appearing with the advent of writing technology some 5 KYA (Hartley 2019; Mullins et al. 2013; Seabright 2010). ${ }^{17}$

The second reason why the characterization of humans as extensive traders is more complex than it might at first appear resides in the fact that many aspects of the major psychological sub-systems underlying their propensity to trade (the disposition and capacity to cooperate reciprocally, and the concepts of property and exchange value) are shared with non-human animals. Given this, seeing humans as standouts for their propensity to trade in fact requires a fine-grained comparative analysis of these sub-systems.

As far as the disposition and capacity to cooperate is concerned, the first thing to note is that humans are not the only organisms that cooperate. Indeed, cooperation is widespread throughout the biological world (Sober and Wilson 1998; West et al. 2011; Sterelny et al. 2013; Griffin et al.). However, this is not to say that there are no differences in the ways that humans and other organisms cooperate. In particular, reciprocal interactions-i.e. where individuals cooperate only as long as others do so, too-are rare outside of the human realm and significantly more restricted (Hammerstein 2003; Hammerstein and Noe 2016; Avsar 2020, p. 16; Cosmides and Tooby 1992). Outside the human sphere, tit-for-tat helping behavior has been observed in vampire bats, chimpanzees, and several other species (Carter and Wilkinson 2013; Hammerstein 2003; Hammerstein and Noe 2016; Ofek 2001). However, there is no question that the extent to which other species rely on reciprocal cooperation is much lower than what is found in humans, where-as just noted-these kinds of interactions can provide the foundation of much their social behavior (Kaplan et al. 2012; Boyd 2018). ${ }^{18}$

When it comes to the concept of property, the first thing to note is that it turns out that Smith (1776) is wrong about the fact that humans are alone in having such a concept (as is Hume 1978 [1739], p. 489, for that matter). Many other animals also recognize property, at least in a rudimentary manner (Kummer 1991; Gintis 2007; Eswaran and Neary 2014; Tibble and Carvalho 2018). For starters, many animals are territorial, and thus recognize that some parts of the environment belong to others (for an overview, see e.g. Hinsch and Komdeur 2017; Sherratt and

\footnotetext{
17 Seabright (2010), in particular, makes clear both the temporal depth of extensive trading and the social and cognitive conditions underlying the reciprocal trust making it possible (including social institutions like money and banking).

18 Indeed, Boyd (2018) argues that in many non-human cases, the conditions making reciprocation mutually profitable seem satisfied, yet there is no reciprocal cooperation. It is also important to emphasize that the fact that humans are standouts for their ability to reciprocally cooperate is not equivalent to them being traders. So, if Cosmides and Tooby (1992) (for example) turn out to be right in arguing that humans evolved dedicated psychological machinery for detecting cheaters in situations of social exchanges, then this, by itself, does not explain that humans are also standouts for being traders. Social exchange conditionals and the detection of cheaters is much wider than trading in property: humans may be standouts for reasoning with rules of the form "if you receive the benefit, then you pay the cost," but they need not apply these rules to trade with property. I can be good at telling if someone violates the rule "If you have a tattoo, then you climbed the mountain," but I may not be inclined to trade my excess food supplies for your excess climbing gear. So, the fact that humans are especially well equipped for reasoning with social exchange conditionals is only an aspect of the explanation of their being traders-it is not the full such explanation.
} 
Mesterton-Gibbons 2015). Furthermore, various animals-including, most notably, primates - can recognize that they have a claim on things other than territories, too (Kummer and Cords 1991; Torii 1974; Pryor 1981; Brosnan 2011; Lakshminarayanan et al. 2008). Still, it is true is that the human concept of property is significantly more sophisticated than that of non-human animals (Kanngiesser et al. 2020).

In particular, human property concepts are deeper. Non-human animals do not recognize others as having a claim on something, and therefore being able to exclude others from it (Tse 2008, p. 287). Rather, they (a) recognize that others simply have the ability (e.g. physical strength) to exclude others from some things, or (b) they fail to see competition for the resource as worthwhile (Kummer 1991; Scorolli et al. 2018; Gintis 2007; Brosnan 2011). ${ }^{19}$ Animals think of things belonging to someone as things that others de facto will or cannot challenge them for-without any normative (i.e. not purely causal) reason for this status being represented. By contrast, humans represent exactly this: they distinguish theft from gifting or borrowing, precisely on the grounds of whether the claim to the relevant good has changed possessor or not (Tse 2008, p. 287). This is a point that has been studied extensively also developmentally, where it has been found, for example, that even young children recognize a history of use as a key component in establishing ownership conventions (apart from physical excludability) (Friedman et al. 2011, 2013).

Furthermore, the breadth of animal property concepts is limited in relation to human property concepts. Non-human animals recognize a variety of things as potential property - some of them even relatively abstract. They can recognize territories as property, they can recognize foodstuffs as property, and they can even recognize some tokens of exchange as property (Brosnan et al. 2008; Parrish et al. 2013). However, they cannot recognize much more than that as potential property (Kanngiesser et al. 2011; Brosnan 2011). In the human case, by contrast, these limits are very wide: humans recognize "air rights," shares, art works, pets, etc. as ownable. Indeed, it is arguable that the human concept of property has no limits at all-people invent new ownable items on a regular basis (cf. the development of mortgage-backed securities).

Very similar remarks hold for the concept of exchange value. Again, at least some non-human animals also have rudiments of such a concept. For example, a number of primates are willing to exert effort to obtain a token that can be exchanged for a piece of cucumber-but only if others do not get a grape for the same effort (Brosnan et al. 2008; Oberliessen and Kalenscher 2019). Thus, these animals show that they think of cucumbers and grapes as exchangeable (though not on a one-to-one basis). ${ }^{20}$

However, the concept of exchange value is significantly narrower in animals than in humans. Humans can conceive of many different things as comparable with each

\footnotetext{
19 This is obviously so for non-biological entities (like fire) or predators, but also extends to members of their own species.

${ }^{20}$ However, it is not clear if these animals would consider an exchange of a large number of cucumber pieces to be equivalent to one grape. Note also that exchange has not been observed in wild populations, and that it is not clear exactly what the primates in these experiments have learned.
} 
other. Indeed, humans have conceived a universal medium of exchange-moneythat allows them to trade anything for anything. While some animals can use tokens (Brosnan et al. 2008; Parrish et al. 2013), this use is more limited: they learn that they can exchange tokens for food, for example, but there is no evidence that they can learn to exchange tokens for toys or tools, say (Brosnan et al. 2008; Parrish et al. 2013). Humans, though, are not so constrained, and exchange money for toys and tools on a regular basis - as well as for aesthetic experiences (such as concerts or art exhibitions), services (such as transportation), and many other things.

All in all, therefore: while none of the three component abilities that make up the propensity to trade are exclusive to humans, important contrasts between human and non-human animals still exist. In particular, humans have the ability to cooperate reciprocally more extensively and frequently than non-human animals, and they have the abilities to rely on deeper and / or more extensive concepts of property and exchange value.

Hence, putting all of this together, it becomes clear that, when it comes to the distinctively human propensity to trade-i.e. the combination of a sophisticated disposition and capacity to cooperate reciprocally, as well as deeper and / or wider concepts of property and exchange value-two facts need to be explained. (1) Why did humans evolve in such a way that they at least sometimes act on such an extensive propensity to trade? That is, why is it that, in some human societies, a major proportion of social interactions revolve around trade-with people engaging in trade of very many goods with very many others-when this is not the case in nonhuman societies? (b) Why does the distinctively human extensive human propensity to trade, barter, and exchange not always display itself? Why do we not find all humans relying on trade to the same extent? The next section sets out answers to these questions.

\section{The bio-cultural evolution of the human propensity to trade}

In order to explain the unique features of the distinctively human extensive propensity to trade-its existence as well as its only sometime display-I proceed in two steps. First, I bring out the selective pressures on the psychological underpinnings of an extensive propensity to trade in humans. Given this, I then, second, show that there is a distinctive set of socio-cultural circumstances that makes the display of this propensity particularly likely.

Before beginning this analysis, though, it is important to make the starting point clear. Given that many non-human animals engage in trade - if only in a rudimentary manner-common evolutionary biological theorizing suggests that early hominins traded much like closely related non-human animals (such as other great apes). Turning this around, it is plausible to think that humans have engaged in trading for a very long time (Brooks et al. 2018; Ofek 2001; Nowell 2010). Given this, what needs an explanation is why trading underwent a pronounced shift in hominins-from something that is not orders of magnitude different from what we find 
in non-human primates to the heavy dependence on complex markets in places like New York or Hong Kong. Doing this is the goal of the rest of this section. ${ }^{21}$

At the heart of the first step of this explanation is the fact that extensive traderather than limited bartering over few goods or services-is highly adaptive where it can be engaged in (see also Ofek 2001, p. 13; Cosmides and Tooby 1992). If I can only exchange territories with you, entering into trading relations with yourather than fighting over the territories-will be adaptive to the extent that future exchanges of territory with you are adaptive. By contrast, if I can exchange territories for food for weapons, with many different people, then the potential gains from trade increase massively and accrue more quickly (Apicella et al. 2014, pp. 1796, 1804; Basu and Waymire 2006, p. 211; Basu et al. 2009; Hartley 2019; Cosmides and Tooby 1992).

To see this in a bit more detail, assume organisms can choose to offer up a trade $(T)$ for a given resource or to simply take it $(A) .^{22}$ Assume also that taking the resource from an organism that is offering a trade is very likely to lead to success in obtaining the resource, that trying to take the resource from an organism that is also trying to take the resource for themselves will only sometimes lead to success, and finally that offering a trade to an organism similarly offering a trade provides some of the benefits that access to the resource provides-but, since something else needs to be given up for this access, not as many of these benefits as simply taking it. (I return to the reasonableness of these assumptions below.) In that case, the payoff matrix of this interaction will take the shape of the classic Prisoner's Dilemma (the numbers in the table represent fitness or utility values - a point to which I return momentarily) (Table 1):

Consider now a situation where the humans playing this game are aware of both the fact that they are interacting strategically and the nature of the game they are playing (the "rational" form of a strategic interaction). In such a situation, the more goods are conceived as tradeable, the greater the likelihood that this game will be conceived of as infinite. In turn, this implies that the game is more likely to have equilibria other than $A / A$ : as is widely known, finitely repeated instances of this game only have $A$ / $A$ as a Nash Equilibrium in all rounds (see e.g. Binmore 1998). The more different goods can be traded with more different others, the less likely it is that any given round of play is the final round. If an organism can trade territory for many other goods, with many different other organisms, the potential number of interactions is large, and there is much less of a clear end of these interactions that is in sight. This can make many strategies_including $T / T$ throughout_equilibrium strategies in the game.

\footnotetext{
21 Inter alia, what needs to be explained is how (some) humans went from a relatively shallow concept of property (e.g. as laid out in Gintis, 2007) to the deeper one found in some contemporary cultures.

22 Note that the arguments to follow differ from the classic hawk / dove / bourgeois accounts of the evolution of property rights: see e.g. Maynard Smith and Parker (1976), Gintis (2007), Eswaran and Neary (2014), Sherratt and Mesterton-Gibbons (2015), and Guala (2016) (see also Smead \& Forber, forthcoming). The argument here is not whether to respect someone else's property rights, but whether to trade for a resource. Similarly, the issue is not just that of determining when it is beneficial for humans to engage in reciprocal interactions: as noted in 18 above, not all forms of reciprocity concern trade.
} 
Importantly, the substance of this argument is unchanged even if the assumption of the rational nature of the interaction is dropped (so that this strategic interaction is turned into a "biological" one). So, assume that (a) humans happen to follow some strategy (whether it is $T, A$, or some combination thereof), and (b) that they adjust this strategy in accordance with how well it does it relation to alternative strategies they encounter. As is also well known, on this biological version of the above game, organisms that always take will invade a population of traders and go to fixation (Skyrms 1996; Binmore 1998; Alexander 2007). Similarly, it is well-known that organisms playing a wide variety of non-purely-taking strategies-such as trade titfor-tat (TFT) — can invade a population of takers in even finitely iterated versions of the game (Skyrms 1996; Binmore 1998; Alexander 2007; Axelrod 1984; Hilbe et al. 2013).

However, what is key for present purposes is that whether this is possible and how quickly it happens depends on the details of the interaction: the number of rounds in each interaction of the game and the differences in the payoffs. ${ }^{23}$ So, for example, the conditions for TFT to be an evolutionary stable strategy in this game are that (1) $w(T F T, T F T)>w(A, T F T)$, or (2) $w(T F T, T F T)=w(T F T, A)$ and $w(T F T, A)>w(A$, $A$ ), where $w(x, y)$ is the fitness payoff of playing $x$ against $y$ (Alexander 2009). Using the above payoff values for illustrative purposes, this implies that if the trading interaction is repeated for two rounds, $w(T F T, T F T)=4$ and $w(A, T F T)=5$. Hence, in that case, TFT is not an ESS. By contrast, for four rounds, $w(T F T, T F T)=8$ and $w(A, T F T)=7 .{ }^{24}$ Hence, increases in the number of rounds of the interaction leads trading-based TFT to become an ESS. ${ }^{25}$ Importantly, furthermore, increasing the number of goods that are seen as tradable, by the same reasoning as on the rational version of the game above, is tantamount to changing the time-horizon of the interaction. The more goods can be traded for more other goods, the more opportunities there are in engaging in trading relations, and thus, the more likely it is that trading with other traders incurs sufficiently large benefits to make it adaptive relative to just

\footnotetext{
${ }^{23}$ In what follows, I focus on $T$-initiated $T F T$; however, the point generalizes to other non-purely- $A$ based strategies.

${ }^{24}$ For three rounds, the second of the above conditions holds, but the issues are clearer to see if the focus is just on the first condition.

25 This point can be strengthened by considering the dynamics of the situation. If the games lasts two rounds, the average fitness of TFT and $A$ are $w(T F T)=3 p+1$ and $w(A)=3 p+2$, where $p$ is the proportion of TFT traders in the population. The average fitness of the population is $\bar{w}=2 p+2$. Assuming, not unreasonably but here largely for convenience (Alexander, 2009; Skyrms, 1996), that the population distributions change according to the replicator dynamics-where strategies change as a function of how their payoff differs from the average in the population-the rate of change of TFT traders is $\partial(T F T) / \partial$ $\mathrm{t}=(1 p-2) p$, which is negative for all $p \in[0,1]$. By contrast, if the game is played for four rounds, the average fitnesses change to $w(T F T)=7 p+1, w(A)=3 p+4$, and $\bar{w}=4 p^{2}+4$. The rate of change of TFT traders becomes $\partial(T F T) / \partial \mathrm{t}=\left(7 p-4 p^{2}-3\right) p$, which is positive for $p>0.75$. Again, therefore: if the number of iterations of the game increases, trading changes from a strategy that is driven to extinction to one that will spread and take over the population (assuming there are sufficiently many other traders in the population).
} 
Table 1 A trading game

\begin{tabular}{lll}
\hline Player 1/player 2 & Trade $(T)$ & Take $(A)$ \\
\hline Trade $(T)$ & 2,2 & 0,4 \\
Take $(A)$ & 4,0 & 1,1 \\
\hline
\end{tabular}

taking or fighting over the resources-both of which have benefits as well (depending on the costs of fights or taking). ${ }^{26}$

What, though, explains that humans are able to engage in extensive trade like this-i.e. of many different goods and services with many others-when non-human animals are not? The answer to this question lies in the particular social environment in which humans evolved.

This social environment was crucially important for the surviving and thriving of individual humans, but it was also genetically complex (including both kin and nonkin) and epistemically opaque (with others' motivational and cognitive states not being obvious). Given these facts-i.e. given that different members of this kind of environment likely had different intentions and beliefs, which impacted their likelihood of providing or reciprocating help_-keeping track of what others are thinking, what their role in society is, and who is in their inner circle of partners became adaptively highly important (Whiten and Byrne 1997; Humphrey 1986; Sterelny 2003, 2012; Schulz 2018).

In an environment like this, a specific set of memory abilities are highly adaptively important. More specifically, given the biological importance of cooperation for all humans, and given the fact that not all members of society can be depended on to be kin and thus to have direct biological interests in cooperating, keeping track of who cooperated with who in the past was very important for humans (Sterelny 2003, 2012). ${ }^{27}$ Similar points hold for the kind of mindreading and motivational abilities that make reciprocal cooperation possible. Determining what others are thinking (whether they are currently hostile or friendly, say) and being able to hold out for large (and possibly uncertain) future benefits at the cost of forgoing smaller (and possibly certain) present benefits ensured continued access to a major element of human adaptive success - the support of others-and thus had major adaptive value (Hrdy 2011; Sterelny 2003, 2012; Cosmides and Tooby 1992; Klein et al.

\footnotetext{
${ }^{26}$ Indeed, this is related to the well-known point that, under some conditions, the Prisoner's Dilemma can be turned into the "Stag Hunt" (in which cooperation is an equilibrium) (Skyrms, 2004; Alexander, 2007). So, in the present case, if the payoffs of the four-round version of the above game are added together-so that this is treated as a one-shot game-the game indeed becomes a Stag Hunt. An alternative way of putting this point is that, with minimal general trading abilities, the interaction gains $T / T$ as an equilibrium focal point (Leeson et al., 2006). A final point worth noting here is that this model applies best to inter-community trading; within a community, the costs of taking plausibly are often so high as to make it an unattractive option compared to some form of cooperation. However, it remains true that trading becomes a particularly beneficial form of cooperation only when many different goods are seen as tradeable.

27 Note that the exact reasons why humans are as cooperative as they are not fully clear as yet (see e.g. Sterelny et al., 2013; West et al., 2011; Sober \& Wilson, 1998). Intriguingly, it is possible that any selective pressures for being traders also fed back into being selective pressures for being cooperators.
} 
2002; see also Rosati et al. 2006; Stevens and Stephens 2008). In short: the kind of social environments that humans evolved in paves the way for the evolution of some of the psychological abilities associated with an extensive propensity for trade: enhanced mindreading abilities, and an expanded capacity for reciprocal cooperation (see also Whiten and Byrne 1997; Cosmides and Tooby 1992; Hrdy 2011). ${ }^{28}$

Most importantly, though, the specific socio-cultural environment that humans evolved in matters for the uniquely human propensity to trade as it allowed them to bio-culturally evolve the kind of cognitive technology that allows them to rely on deep and wide concepts of (a) property and (b) exchange value, and (c) to engage in widespread reciprocal cooperation.

To see this, note that humans are extensive cultural learners. Without the guidance and information of their conspecifics, even their basic survival would be difficult (Heyes 2018; Boyd and Richerson 2005; Henrich 2015; Henrich and McElreath 2007; Tomasello 1999; Sterelny 2003, 2012; Legare 2017). This human ability to rely on cultural learning is crucial in this context, as it also allows humans to be sophisticated tool builders and tool users (Biro et al. 2013; Tennie and Over 2012; van Schaik and Pradhan 2003; Vaesen 2012; Henrich 2015; Boyd and Richerson 2005; Landy et al. 2014; Osiurak and Reynaud 2020; Muthukrishna and Henrich 2016).

While many different kinds of animals (see e.g. Mann and Patterson 2013; Haslam 2013; Sanz and Morgan 2013; Fragaszy et al. 2013; McGrew 2013; Hansell and Ruxton 2008; Cheke et al. 2011; Shumaker et al. 2011) have been shown to use or manufacture tools, humans are able to build and use tools of particularly high degrees of complexity (Shea 2017). Given their high degrees of complexity, the manufacture and use of these tools is very difficult for individuals, but can be achieved through cultural learning. Learning from scratch how to make human tools like Neolithic weapons is difficult for a single human: it requires the selection, collection, and shaping of the right materials, generally over long periods of time, as well as much practice in using these materials. If individuals had to rediscover how to do this every generation, the probability of the emergence of sophisticated technology in the human lineage would be small. However, if tools can be refined cumulatively over generations - through cultural learning - this probability increases drastically (Boyd and Richerson 2005; Boyd et al. 2011).

Importantly furthermore, this point goes beyond the manufacture and use of sophisticated physical tools-swords and ploughshares-but extends to sophisticated cognitive technology as well (Vaesen 2012; Dennett 2000; Osiurak and Reynaud, forthcoming; Schulz 2020). In particular, through cultural learning, humans became able to construct and use advanced mnemonic and inferential tools: quipus, paper, calculating devices like the "Senkereh Tablet" (Sugden 1981) and sundials (King 1955), written language (Basu and Waymire 2006; Basu et al. 2009; Tse 2008; Hartley 2019; Mullins et al. 2013), myths, songs, dances, and algebraic conventions. These devices allowed humans to store and process more and more complex information more efficiently and for longer time periods, and thus vastly enhanced their

${ }^{28}$ Field (2002) notes that this point is missing in Ofek (2001). 
cognitive abilities in certain dimensions. In particular, there are two aspects in which the ability to forge cognitive technology greatly altered human thought and action.

First, cognitive tools enabled humans to often rely on many highly complex and abstract concepts in their decision making (Landy et al. 2014; Stout and Chaminade 2012; Muthukrishna and Henrich 2016; Schulz 2020). Highly complex and abstract concepts do not have a clear empirical signature: there is no easily observable set of features that all causes or all cases of justice have in common. Hence, applying these kinds of concepts requires much time, concentration, and attention (Fodor 1983, 1990; Prinz 2002; Margolis and Laurence 2015). Without cognitive tools, the frequent use of many highly complex and abstract concepts would thus generally be impossible or not worthwhile.

Note that this is not to say that the use of some highly complex and abstract concepts (like [CAUSE] or [NUMBER]) do not have an innate foundation as well (Carey and Spelke 1996; de Hevia et al. 2014; Gopnik et al. 2004). Rather, the claim is that, whatever else may be needed to rely on many highly complex and abstract concepts, without the uses of cognitive technology, this reliance is unlikely to be possible. The storage of important, frequently used information-e.g. using quipus or writing - frees concentration and attention: organisms do not have to use scarce cognitive resources (including time) to recall that information, but can simply look it up. Complex representational inferences are made easier if intermediate steps of the inference can be written down or if parts of it can be automated (e.g. using computational devices like sundials) (Schulz 2018, 2020).

So, for example, determining if there is a pattern in the occurrence of certain types of events - that is, applying concepts like [REGULAR] [PERIODICITY] to the world-is very hard: the events can be spaced far apart from each other in space or time, and the detection of their underlying periodicity may require the consultation of a large number of instances of the relevant type of events. However, if the events can be tracked and analyzed with cognitive tools like written records and a number system that allows for the calculation of averages, the detection of patterns in them becomes much easier; indeed, it is not clear that it would be possible without this (Gibson and Ingold 1993; Mullins et al. 2013). For a concrete example, consider determining whether the "Pharaoh cicadas" Magicicada septendecim emerge randomly or with a regular periodicity. It turns out that doing so requires tracking the cicadas over several decades - the cicadas emerge every 17 years - which would be very difficult for humans without the aid of cognitive technology (see also Kelly 2015 , for more on the amounts of information that can be stored and tracked in nonliterate societies).

Noting this is key for present purposes, as the concepts of property and exchange value-especially in their deeper and wider forms-are abstract and complex in exactly this way. These concepts are built out of other concepts like [CLAIM], [EXCLUDABLE], [MEANS], [EXCHANGE], and they do not have a straightforward perceptual signature: many different states of the world can constitute A's owning something. Because of this, reliance on such concepts is highly time-, concentration-, and attention-consuming (Schulz 2018, 2020).

This is important, as it implies that, in the absence of tools that help relate these concepts to the actual conditions prevailing in the world, having and using such 
concepts is unlikely to even be possible (Schulz 2020). It is only though cognitive tools like quipus, petroglyphs, myths, songs, writing, or algebra that it becomes possible for humans to recognize highly abstract things as belonging to someone, and they can come to recognize the excludability of an owned entity as resting on abstract notions like [DESERT], [HISTORICAL CLAIMS], or [MIXING OF LABOR] (Scorolli et al. 2018; see also Ofek 2001, pp. 180-187; Hartley 2019; Mullins et al. 2013). Recognizing that person A owns object P because A, decades ago, mixed their labor in the appropriate manner with $\mathrm{P}$ (say), and that therefore, A deserves to decide what to do with $\mathrm{P}$ even if $\mathrm{A}$ is not in direct physical contact with $\mathrm{P}$ is very difficult without tools that allow us to store what A did decades ago, to compare this to what A needed to do to acquire a claim on $\mathrm{P}$, to transmit what A intends to do with $\mathrm{P}$ even where $\mathrm{P}$ is physically far apart from A, etc. Cognitive technology is needed to tie these highly abstract and complex concepts to the actual conditions prevailing in the environment at any given point in time. ${ }^{29}$

Importantly, though, since humans are cultural learners, they can obtain exactly these kinds of sophisticated cognitive tools. Hence, it is in particular humans that are able to rely on deeper and / or wider concepts of property and exchange value in their interactions with each other.

The second aspect of the importance of cognitive technology is that it supports more sophisticated reciprocal cooperation. Keeping track of who helped who when and how is easier - and may even be made possible in the first place-when this information can be stored externally and recalled when needed. Doing this frees up cognitive resources like concentration, attention, and memory (Mullins et al. 2013). In the absence of this technology, sophisticated reciprocal cooperation may not be practically feasible: with many different goods that can be traded with many different others, the mnemonic and processing demands of tracking trades quickly become so vast as to make the latter practically impossible without relying on cognitive technology like writing, algebra, or quipus.

Putting all of this together: in order for a species to be able to be trade with many different goods - and thus, to harness the adaptive benefits of extensive trade-cognitive technology is crucial. Once many things are seen as tradable with many others, it becomes vital to have ways of easily checking who currently owns what, and who got what from who. Cognitive technology (a quipus, petroglyphs, written language, algebra, computational devices, etc.) allows exactly this. In turn, in order to acquire such a technology, though, cultural learning is crucial. Such technology is highly unlikely to be discovered by individual organisms, but needs to be refined over generations (Tomasello 1999; Sterelny 2012; Heyes 2018). This, thus, explains why humans - unlike other organisms - evolved the ability to be extensive traders: humans, more than other animals, evolved in a particular complex social and cultural environment that provided the ground for the acquisition of the cognitive technology needed to support a sophisticated disposition and capacity for reciprocity

\footnotetext{
29 Again, this is not to say that underlying these concepts are not further innate abilities-e.g. concerning mindreading or future planning (Kabadayi \& Osvath, 2017; Leslie et al., 2004). The point is just that, whatever else is needed to use these abilities, cognitive tools are necessary, too.
} 
as well as deep and / or wide concepts of property and exchange value-and thus, which can underwrite large-scale trading relations.

This, though, answers only one of the two questions that are open concerning the extensive propensity to trade found in humans. We also need to explain why this extensive propensity does not always display itself. Why do not all human populations engage in extensive trade? What accounts for the variability in this respect?

Given the above, though, the outline of the answer to this question can now be easily seen. In particular, this answer turns on the fact that the extensive propensity to trade comes to its own only if it is supported by the right external arrangements. Specifically, humans can rely on deeper and / or wider concepts of property and exchange value to the extent that the relevant cognitive technology is available. Where humans do not have access to this kind of technology, they are also less likely to rely on deeper and / or wider concepts of property and exchange value, and are less likely to be able to engage in the trade with many different goods. ${ }^{30}$

Importantly, though, the appropriate kind of cultural evolution need not occur in all cultures. Not all cultures have evolved the same kinds of cognitive technologyfor example, fairly few cultures have a powerful cognitive technology like a written language, and quipus, too, are not found in every culture. Cultural evolution is not a deterministic system, but comes with much variability: differences in who learns from who and in what manner can lead to very different cultural outcomes (Henrich 2015, 2020; Henrich and McElreath 2007; Heyes 2018; Tomasello 1999). Therefore, not all cultures may have the technological underpinnings to allow for sophisticated reciprocal cooperation and especially deep and / or wide concepts of property and exchange value. Ipso facto, not all cultures will display the propensity to trade to the same extent (or at all).

Turning this around: where cultural learning created the cognitive technology that supports relatively strong reciprocal cooperation, and relatively deep and / or extensive concepts of property and exchange value, it was possible to trigger an extensive propensity to trade-i.e. one that allowed for trade among a wide number of goods with a wide number of people. In this way, it becomes possible to see why it is both true that extensive trade is an important part of the human lineage (see e.g. Ofek 2001 , pp. 136, 172, 217), and that trade is culturally and temporally specific (see e.g. Apicella et al. 2014; Avsar 2020, pp. 16-17; see also Eswaran and Neary 2014).

To understand this better, it is important to forestall some possible misconceptions. First of all, the claim here is not that trade in the human lineage came into existence with the development of cognitive technology. In fact, there is every reason to think that trading has been around longer than the kinds of cognitive technology (algebraic conventions, a written language, etc.) just mentioned (Nowell 2010; Ofek 2001). Rather, the argument here is that the particularly extensive propensity to trade found in some humans is associated with the advent of this kind of cognitive technology. Undoubtedly, as noted earlier, some humans traded before they

\footnotetext{
30 Again, Seabright (2010) underscores this point well, noting that extensive human cooperation needs to be underwritten by social institutions (like money) that scaffold trusting relations among strangers.
} 
culturally evolved this kind of technology; however, a uniquely strong propensity to trade came later once humans had culturally evolved this kind of technology.

Importantly, this is also a key prediction that the present account makes: we should find a correlation between the cultural evolution of the above kinds of cognitive tools and the emergence of extensive trading relations. As it turns out, though further corroboration of this prediction would be useful, there is some initial support for this prediction. For example, extensive trading institutions tend to be seen only where a culture has developed writing technology, such as the Mayan, Babylonian, ancient Egyptian, and ancient Chinese ones (Hartley 2019; Mullins et al. 2013). ${ }^{31}$ At this stage, though, this first prediction is at least to be seen as credible.

Second and relatedly, the claim is also not that humans did not cooperate extensively before they developed this kind of cognitive technology. Again, there is every reason to think that this is false (Cosmides and Tooby 1992; Sterelny 2003, 2012). Rather, the point is that the extensive reciprocal cooperation that is characteristic of trade with many different goods and many different people is tied to the cultural emergence of cognitive technology.

In this way, the two questions from the previous section can be answered. (1) The reason why humans evolved in such a way that they sometimes display an extensive propensity to trade lies in the fact that they sometimes lived in the kinds of socio-cultural environments that provided conditions for the bio-cultural evolution of the cognitive tools needed to underwrite a sophisticated disposition and capacity to cooperate, and deeper and / or wider concepts of property and exchange value. (2) This human propensity to trade does not always display itself, as it requires the right institutional settings to be triggered-namely, the existence of cognitive technology like writing that can support many repeated reciprocal interactions, as well as deep and / or wide concepts of property and exchange value.

\section{Conclusion}

How do humans differ from other animals in their propensity to trade? Why does this propensity not always display itself even among humans? This paper developed an integrated set of answers to these two questions. The propensity to trade breaks down into three component psychological traits: the ability to cooperate, and concepts of property and exchange value. At the core of the human/non-human differences in these three traits is the fact that, due the fact that humans evolved in a specific kind of socio-cultural environment, they evolved a more extensive ability to interact reciprocally, as well as the ability to rely on especially deep and / or wide concepts of property and exchange value. In turn, these differences allowed humans

\footnotetext{
31 Note also that it is not clear that other distinguishing features of these cultures-such as their large range-are a plausible explanation of them being based on extensive trade. It's a classic economic insight that, even in small-scale cultures, there is much gain from specialization and division of labor (as seen in the classic works of Ricardo and Smith). Hence, the fact that these cultures span different geographical ranges is, if anything, better seen as the outcome of their containing extensive trading and cognitive technology, rather than presupposition of the latter.
} 
to display an extensive propensity to trade in the right institutional settings: namely, ones where the appropriate cognitive technology culturally evolved that allowed many different things to be traded for many other things with many other people.

\section{References}

Alexander J (2007) The structural evolution of morality. Cambridge University Press, Cambridge

Alexander J (2009) Evolutionary game theory. In: Zalta EN (ed) The stanford encyclopedia of philosophy. http://plato.stanford.edu/archives/fall2009/entries/game-evolutionary/

Apicella CL, Azevedo EM, Christakis NA, Fowler JH (2014) Evolutionary origins of the endowment effect: evidence from hunter-gatherers. Am Econ Rev 104(6):1793-1805. https://doi.org/10.1257/ aer.104.6.1793

Avsar R (2020) The evolutionary origins of markets: how evolution, psychology, and biology have shaped the economy. Routledge, London

Axelrod R (1984) The evolution of cooperation. Basic Books, New York

Basu S, Waymire GB (2006) Recordkeeping and human evolution. Acc Horizons 20(3):201-229. https:// doi.org/10.2308/acch.2006.20.3.201

Basu S, Dickhautb J, Hecht G, Towry K, Waymire GB (2009) Recordkeeping alters economic history by promoting reciprocity. Proc Natl Acad Sci USA 106(4):1009-1014

Binmore K (1998) Game theory and the social contract, Vol II: Just playing. MIT Press, Cambridge

Binmore K (2007) Playing for real. Oxford University Press, Oxford

Biro D, Michael H, Rutz C (2013) Tool use as adaptation. Philos Trans R Soc B 368(1630):20120408. https://doi.org/10.1098/rstb.2012.0408

Blake PR, Harris PL (2009) Children's understanding of ownership transfers. Cogn Dev 24(2):133-145. https://doi.org/10.1016/j.cogdev.2009.01.002

Boyd R (2018) A different kind of animal: how culture transformed our species. Princeton University Press, Princeton

Boyd R, Richerson P (2005) The origin and evolution of cultures. Oxford University Press, Oxford

Boyd R, Richerson P, Henrich J (2011) The cultural niche: Why social learning is essential for human adaptation. Proc Natl Acad Sci USA 108(Supplement 2):10918-10925. https://doi.org/10.1073/ pnas. 1100290108

Brooks AS, Yellen JE, Potts R, Behrensmeyer AK, Deino AL, Leslie DE, Ambrose SH, Ferguson JR, d'Errico F, Zipkin AM, Whittaker S, Post J, Veatch EG, Foecke K, Clark JB (2018) Long-distance stone transport and pigment use in the earliest Middle Stone Age. Science 360(6384):90-94. https://doi.org/10.1126/science.aao2646

Brosnan SF (2011) Property in Nonhuman primates. New Directions Child Adolesc Dev 132:9-22. https://doi.org/10.1002/cd.293

Brosnan SF, Beran MJ (2009) Trading behavior between conspecifics in chimpanzees. J Comp Psychol 123(2):181-194

Brosnan SF, Jones OD, Lambeth SP, Mareno MC, Richardson AS, Schapiro SJ (2007) Endowment effects in chimpanzees. Curr Biol 17:1-4

Brosnan SF, Grady MF, Lambeth SP, Schapiro SJ, Beran MJ (2008) Chimpanzee autarky. PLoS ONE 3:e1518

Brosnan SF, Jones OD, Gardner M, Lambeth SP, Schapiro SJ (2012) Evolution and the expression of biases: situational value changes the endowment effect in chimpanzees. Evol Hum Behav 33:378-386

Carey S, Spelke E (1996) Science and core knowledge. Philos Sci 63:515-533

Carter G, Wilkinson G (2013) Food sharing in vampire bats: reciprocal help predicts donations more than relatedness or harassment. Proc R Soc B 280:20122573

Cheke LG, Bird CD, Clayton NS (2011) Tool-use and instrumental learning in the Eurasian jay (Garrulus glandarius). Anim Cogn. https://doi.org/10.1007/s10071-011-0379-4

Cosmides L, Tooby J (1992) Cognitive adaptations for social exchange. In: Barkow J, Cosmides L, Tooby $\mathrm{J}$ (eds) The adapted mind: evolutionary psychology and the generation of culture. Oxford University Press, Oxford, pp 163-228 
de Hevia MD, Izard V, Coubart A, Spelke ES, Streri A (2014) Representations of space, time, and number in neonates. Proc Natl Acad Sci USA 111:4809-4813

Dennett DC (2000) Making tools for thinking. In: Sperber D (ed) Metarepresentation: a multidisciplinary perspective. Oxford University Press, Oxford, pp 17-29

Drayton LA, Brosnan SF, Carrigan J, Stoinski TS (2013) Endowment effect in gorillas (Gorilla gorilla). J Comp Psychol 127:365-369

Eswaran M, Neary HM (2014) An economic theory of the evolutionary emergence of property rights. Am Econ J 6(3):203-226. https://doi.org/10.2307/43189681

Field A (2002) Review of Ofek (2001)—Second Nature. J Econ Hist 62(03):922-924

Flemming TM, Jones OD, Mayo L, Stoinski T, Brosnan SF (2012) The endowment effect in orangutans. Int J Comp Psychol 25:285-298

Fodor J (1983) The modularity of mind: an essay on faculty psychology. MIT Press, Cambridge

Fodor J (1990) The theory of content. MIT Press, Cambridge

Fragaszy DM, Biro D, Eshchar Y, Humle T, Izar P, Resende B, Visalberghi E (2013) The fourth dimension of tool use: temporally enduring artefacts aid primates learning to use tools. Philos Trans $\mathrm{R}$ Soc B 368(1630):20120410. https://doi.org/10.1098/rstb.2012.0410

Friedman O, Neary KR, Defeyter MA, Malcolm SL (2011) Ownership and object history. New Directions Child Adolesc Dev 2011(132):79-89. https://doi.org/10.1002/cd.298

Friedman O, Van de Vondervoort JW, Defeyter MA, Neary KR (2013) First possession, history, and young children's ownership judgments. Child Dev 84(5):1519-1525. https://doi.org/10.1111/cdev. 12080

Gibson KR, Ingold T (eds) (1993) Tools, language and cognition in human evolution. Cambridge University Press, Cambridge

Gintis H (2007) The evolution of private property. J Econ Behav Organ 64:1-16

Gopnik A, Glymour C, Sobel DM, Schulz LE, Kushnir T, Danks D (2004) A theory of causal learning in children: causal maps and Bayes nets. Psychol Rev 111(1):3-32. https://doi.org/10.1037/0033295X.111.1.3

Griffin AS, West SA, Buckling A (2004) Cooperation and competition in pathogenic bacteria. Nature 430:1024-1027

Guala F (2016) Understanding institutions: the science and philosophy of living together. Princeton University Press, Princeton

Hammerstein P (2003) Why is reciprocity so rare in social animals? A protestant appeal. In: Hammerstein P (ed) Genetic and cultural evolution of cooperation. MIT Press, Cambridge, pp 83-94

Hammerstein P, Noe R (2016) Biological trade and markets. Philos Trans R Soc B. https://doi.org/10. 1098/rstb.2015.0101

Hansell M, Ruxton GD (2008) Setting tool use within the context of animal construction behaviour. Trends Ecol Evol 23(2):73-78. https://doi.org/10.1016/j.tree.2007.10.006

Hartley T (2019) The continuing evolution of ownership. PLoS ONE 14(2):e021187

Haslam M (2013) 'Captivity bias' in animal tool use and its implications for the evolution of hominin technology. Philos Trans R Soc B 368(1630):20120421. https://doi.org/10.1098/rstb.2012.0421

Hausman DM (1992) The inexact and separate science of economics. Cambridge University Press, Cambridge

Hausman DM (2012) Preference, value, choice, and welfare. Cambridge University Press. https://books. google.com/books?id=tqLZ13emsB8C

Henrich J (1997) Market incorporation, agricultural change, and sustainability among the Machiguenga Indians of the Peruvian Amazon. Hum Ecol 25(2):319-351

Henrich J (2015) The secret of our success: how culture is driving human evolution, domesticating our species, and making us smarter. Princeton University Press, Princeton

Henrich J, McElreath R (2007) Dual-inheritance theory: the evolution of human cultural capacities and cultural evolution. In: Dunbar R, Barrett L (eds) The Oxford handbook of evolutionary psychology. Oxford University Press, pp 555-570

Henrich J, Boyd R, Bowles S, Camerer C, Fehr E, Gintis H, McElreath R (2001) In search of homo economicus: behavioral experiments in 15 small-scale societies. Am Econ Rev 91(2):73-78

Henrich J (2020) The WEIRDest people in the world. Farrar, Straus and Giroux

Heyes CM (2018) Cognitive gadgets: the cultural evolution of thinking. Harvard University Press, Cambridge

Hilbe C, Nowak MA, Sigmund K (2013) Evolution of extortion in iterated prisoner's dilemma games. PNAS 110(17):6913-6918 
Hinsch M, Komdeur J (2017) What do territory owners defend against? Proc R Soc B. https://doi.org/10. $1098 /$ rspb.2016.2356

Hrdy SB (2011) Mothers and others: the evolutionary origins of mutual understanding. Harvard University Press, Cambridge

Hume D (1978 [1739]) A treatise of human nature. In Selby-Bigge, LA, Nidditch PH (eds) Clarendon Press, Oxford

Humphrey N (1986) The inner eye: social intelligence in evolution. Oxford University Press, Oxford

Jaeger CB, Brosnan SF, Levin DT, Jones OD (2020) Predicting variation in endowment effect magnitudes. Evol Hum Behav 41(3):253-259. https://doi.org/10.1016/j.evolhumbehav.2020.04.002

Jones OD (2001) Time-shifted rationality and the law of law's leverage: behavioral economics meets behavioral biology. Northwest Univ Law Rev 95:1141-1205

Kabadayi C, Osvath M (2017) Ravens parallel great apes in flexible planning for tool-use and bartering. Science 357(6347):202-204. https://doi.org/10.1126/science.aam8138

Kanngiesser P, Santos LR, Hood BM, Call J (2011) The limits of endowment effects in great apes (Pan paniscus, Pan troglodytes, Gorilla gorilla, Pongo pygmaeus). J Comp Psychol 125:436-445

Kanngiesser P, Rossano F, Frickel R, Tomm A, Tomasello M (2020) Children, but not great apes, respect ownership. Dev Sci 23(1):e12842. https://doi.org/10.1111/desc.12842

Kaplan HS, Schniter E, Smith VL, Wilson BJ (2012) Risk and the evolution of human exchange. Proc Biol Sci 279(1740):2930-2935. https://doi.org/10.1098/rspb.2011.2614

Kelly L (2015) Knowledge and power in prehistoric societies: orality, memory and the transmission of culture. Cambridge University Press, Cambridge

King HC (1955) The history of the telescope. Charles Griffin \& Co., London

Klein SB, Cosmides L, Tooby J, Chance S (2002) Decisions and the evolution of memory: multiple systems, multiple functions. Psychol Rev 109(2):306-329

Kummer H (1991) Evolutionary transformations of possessive behavior. J Soc Behav Pers 6:75-83

Kummer H, Cords M (1991) Cues of ownership in long-tailed macaques, Macaca fascicularis. Anim Behav 42:529-549

Lakshminarayanan V, Chen MK, Santos LR (2008) Endowment effect in capuchin monkeys. Philos Trans R Soc Lond b 363:3837-3844

Landy D, Allen C, Zednik C (2014) A perceptual account of symbolic reasoning. Front Psychol 5:275. https://doi.org/10.3389/fpsyg.2014.00275

Leeson PT, Coyne CJ, Boettke PJ (2006) Converting social conflict: focal points and the evolution of cooperation. Rev Austrian Econ 19:137-147. https://doi.org/10.1007/s11138-006-7345-4

Legare CH (2017) Cumulative cultural learning: development and diversity. Proc Natl Acad Sci USA 114(30):7877-7883

Leslie AM, Friedman O, German TP (2004) Core mechanisms in 'theory of mind.' Trends Cogn Sci 8(12):528-533

Locke J (1987 [1866]) The two treatises of civil government (R. Ashcraft, Ed.). Routledge, London

Mann J, Patterson EM (2013) Tool use by aquatic animals. Philos Trans R Soc B. https://doi.org/10.1098/ rstb.2012.0424

Margolis E, Laurence S (eds) (2015) The conceptual mind. MIT Press, Cambridge

Maynard Smith J, Parker GA (1976) The logic of asymmetric contests. Anim Behav 24:159-175

McGrew WC (2013) Is primate tool use special? Chimpanzee and New Caledonian crow compared. Philos Trans R Soc B 368(1630):422. https://doi.org/10.1098/rstb.2012.0422

Mulcahy NJ, Call J (2006) Apes save tools for future use. Science 312(5776):1038-1040. https://doi.org/ $10.1126 /$ science. 1125456

Mullins DA, Whitehouse H, Atkinson QD (2013) The role of writing and recordkeeping in the cultural evolution of human cooperation. J Econ Behav Organ 90S:S141-S151

Muthukrishna M, Henrich J (2016) Innovation in the collective brain. Philos Trans R Soc B. https://doi. org/10.1098/rstb.2015.0192

Noe R, Voelkl B (2013) Cooperation and biological markets: the power of partner choice. In: Sterelny K, Joyce R, Calcott B, Fraser B (eds) Cooperation and its evolution. MIT Press, Cambridge, pp $131-152$

Nowak MA, Sigmund K (2005) Evolution of indirect reciprocity. Nature. https://doi.org/10.1038/natur e04131

Nowell A (2010) Defining behavioral modernity in the context of neandertal and anatomically modern human populations. Annu Rev Anthropol 39(1):437-452. https://doi.org/10.1146/annurev.anthro. 012809.105113 
Oberliessen L, Kalenscher T (2019) Social and non-social mechanisms of inequity aversion in nonhuman animals. Front Behav Neurosci 13:133. https://doi.org/10.3389/fnbeh.2019.00133

Ofek H (2001) Second nature: economic origins of human evolution. Cambridge University Press, Cambridge

Ofek H (2013) MHC-mediated benefits of trade: a biomolecular approach to cooperation in the marketplace. In: Sterelny K, Joyce R, Calcott B, Fraser B (eds) Cooperation and its evolution. MIT Press, Cambridge, pp 175-194

Osiurak F, Reynaud E (2020) The elephant in the room: what matters cognitively in cumulative technological culture. Behav Brain Sci. https://doi.org/10.1017/S0140525X19003236

Parrish AE, Perdue BM, Evans TA, Beran MJ (2013) Chimpanzees (Pan troglodytes) transfer tokens repeatedly with a partner to accumulate rewards in a self-control task. Anim Cogn 16(4):627-636. https://doi.org/10.1007/s10071-013-0599-x

Pelé M, Dufour V, Thierry B, Call J (2009) Token transfers among great apes (Gorilla gorilla, Pongo pygmaeus, Pan paniscus, and Pan troglodytes): species differences, gestural requests, and reciprocal exchange. J Compr Psychol 123(4):375-384. https://doi.org/10.1037/a0017253

Prinz J (2002) Furnishing the mind: concepts and their perceptual basis. MIT Press, Cambridge

Pryor FL (1981) A survey of the economic systems of wild chimpanzees and baboons. J Econ Issues 15(1):33-59

Rosati AG, Stevens JR, Hauser MD (2006) The effect of handling time on temporal discounting in two New World primates. Anim Behav 71:1379-1387

Sanz CM, Morgan DB (2013) Ecological and social correlates of chimpanzee tool use. Philos Trans R Soc B 368(1630):20120416. https://doi.org/10.1098/rstb.2012.0416

Schliesser E (2011) Reading Adam Smith after Darwin: On the evolution of propensities, institutions, and sentiments. J Econ Behav Org 77(1):14-22. https://doi.org/10.1016/j.jebo.2010.04.007

Schulz A (2011) Sober \& Wilson's evolutionary arguments for psychological altruism: a reassessment. Biol Philos 26:251-260

Schulz A (2018) Efficient cognition: the evolution of representational decision making. MIT Press, Cambridge

Schulz A (2020) Enhancing thoughts: culture, technology, and the evolution of human cognitive uniqueness. Mind \& Language. https://doi.org/10.1111/mila.12320

Scorolli C, Borghi AM, Tummolini L (2018) Cues of control modulate the ascription of object ownership. Psychol Res 82:929-954. https://doi.org/10.1007/s00426-017-0871-9

Seabright P (2010) The company of strangers: a natural history of economic life, Revised. Pricenton University Press, Pricenton

Shea JJ (2017) Stone tools in human evolution: behavioral differences among technological primates. Cambridge University Press, Cambridge

Sherratt TN, Mesterton-Gibbons M (2015) The evolution of respect for property. J Evol Biol 28:1185-1202

Shumaker, B. R. W., Walkup, K. R., \& Beck, B. B. (2011). Animal Tool Behavior: The Use and Manufacture of Tools by Animals (Revised and Updated ed.). Johns Hopkins University Press.

Skyrms B (1996) Evolution and the social contract. Cambridge University Press, Cambridge

Skyrms B (2004) The stag hunt and the evolution of social structure. Cambridge University Press

Smead R, Forber P (forthcoming) Convention and the origins of ownership. Philos Sci. https://doi.org/10. $1086 / 710626$

Smith A (1776) An inquiry into the nature and causes of the wealth of nations

Sober E, Wilson DS (1998) Unto others: the evolution and psychology of unselfish behavior. Harvard University Press, Cambridge, MA

Sterelny K (2003) Thought in a hostile world: the evolution of human cognition. Wiley-Blackwell, Malden

Sterelny K (2012) The evolved apprentice: how evolution made humans unique. MIT Press, Cambridge

Sterelny K, Joyce R, Calcott B, Fraser B (eds) (2013) Cooperation and its evolution. MIT Press, Cambridge

Stevens JR, Stephens DW (2008) Patience. Curr Biol 18:R11-12

Stich S (2007) Evolution, altruism and cognitive architecture: a critique of Sober and Wilson's argument for psychological altruism. Biol Philos 22:267-281

Stout D, Chaminade T (2012) Stone tools, language and the brain in human evolution. Philos Trans R Soc Lond B 367(1585):75-87 
Sugden KF (1981) A history of the abacus. Acc Hist J 8(2):1-22. https://doi.org/10.2308/0148-4184.8. 2.1

Tennie C, Over H (2012) Cultural intelligence is key to explaining human tool use. Behav Brain Sci 35(4):242-243. https://doi.org/10.1017/S0140525X11001968

Tibble L, Carvalho S (2018) Rethinking the evolution of property and possession: a review and methodological proposition. Evol Anthropol 27(6):285-296. https://doi.org/10.1002/evan.21748

Tomasello M (1999) The cultural origins of human cognition. Harvard University Press, Cambridge

Torii, M. (1974). Possession by non-human primates. In: Campbell B (ed) Contemporary primatology, 310-314

Triki Z, Wismer S, Rey O, Ann Binning S, Levorato E, Bshary R (2019) Biological market effects predict cleaner fish strategic sophistication. Behav Ecol 30(6):1548-1557. https://doi.org/10.1093/beheco/ $\operatorname{arz} 111$

Tse PU (2008) Symbolic thought and the evolution of human morality. In: Sinnott-Armstrong W (ed) Moral psychology. Vol 1-The Evolution of Morality Adaptations and Innateness. MIT Press, Cambridge, pp 269-297

Vaesen K (2012) The cognitive bases of human tool use. Behavioral and Brain Sciences 35(4):203-218

van Schaik CP, Pradhan GR (2003) A model for tool-use traditions in primates: implications for the coevolution of culture and cognition. J Hum Evol 44(6):645-664

West SA, El Mouden C, Gardner A (2011) Sixteen common misconceptions about the evolution of cooperation in humans. Evol Hum Behav 32(4):231-262. https://doi.org/10.1016/j.evolhumbehav.2010. 08.001

Whiten A, Byrne RW (eds) (1997) Machiavellian intelligence II: extensions and evaluations. Cambridge University Press, Cambridge

Wilson DS, Gowdy JM, Rosser JB Jr (2013) Rethinking economics from an evolutionary perspective. J Econ Behav Organ 90S:S1-S2

World Bank. Trade (\% of GDP). Retrieved August 3 from https://data.worldbank.org/indicator/NE.TRD. GNFS.ZS

Publisher's Note Springer Nature remains neutral with regard to jurisdictional claims in published maps and institutional affiliations. 